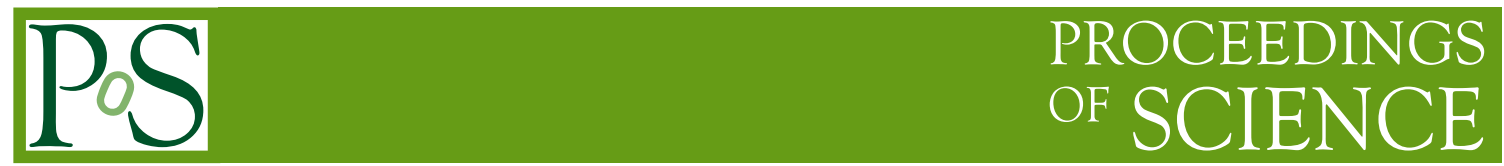

\title{
A brief review of Leptogenesis
}

\author{
Marta Losada* \\ Centro de Investigaciones en Ciencias Basicas y Aplicadas \\ Universidad Antonio Nariño \\ Bogotá, Colombia \\ E-mail: malosada@uan.edu.co
}

In this talk I briefly review main issues in leptogenesis which are relevant for the quantitative calculation of the final baryon asymmetry of the Universe, focusing on diverse lepton flavour effects.

35th International Conference of High Energy Physics - ICHEP2010, July 22-28, 2010

Paris France

${ }^{*}$ Speaker. 


\section{Introduction}

Leptogenesis is a viable mechanism that provides an answer to the observed matter-antimatter asymmetry of the Universe[1],[2]. A final lepton asymmetry is produced by the asymmetric decay of a heavy particle to lepton and antileptons, which is then converted into a baryon asymmetry through sphaleron interactions. The heavy particle must decay out-of-equilibrium in a CP-violating way, thus satisfying all of the conditions established long ago by Sakharov [3]. One of the many advantages of leptogenesis is that it is occuring in the same framework that generates neutrino masses. Thus contributing simultaneously to the explanation of two important experimental observations. However, the main drawback to leptogenesis is precisely that it is very hard to verify experimentally.

The generic characteristics that are required for the leptogenesis mechanism to work are:

- to have mixing/interference between (at least) two different states. Typically flavour, CP eigenstates, etc.

- in general all possible mechanisms of $\mathrm{CP}$ violation can be implemented

- the careful consideration of the background dynamics of the expanding Universe

- the relevance of B and B-L conserving processes to determine the asymmetric densities of the different species

- the explicit incorporation of $\mathrm{L}, \mathrm{C}$ and $\mathrm{CP}$ violation is model dependent.

Keeping these features in mind there is a plethora of possible models such as [2]: Standard Model (SM) + see-saw [4] (with 3 hierarchical right-handed neutrinos) and the corresponding supersymmetric version; Resonant leptogenesis, Soft Leptogenesis, Dirac Leptogenesis, Scalar/Fermion Triplet Leptogenesis, Electromagnetic Leptogenesis, etc.

In the minimal model the Lagrangian is given by:

$$
\mathscr{L}_{N P}=\lambda_{\alpha i} \ell_{\alpha} \phi N_{i}+\text { h.c. }+M_{i} N_{i} N_{i}
$$

An initial lepton asymmetry is generated in the out-of-equilibrium decays of heavy singlet Majorana neutrinos, and is then partially converted in a baryon asymmetry by anomalous sphaleron interactions [5]. Heavy Majorana singlet neutrinos are also a fundamental ingredient of the seesaw model [4], that provides an elegant explanation of the suppression of neutrino masses with respect to all other Standard Model (SM) mass scales. In the case of SM + see-saw, a relevant bound is placed on the net CP-asymmetry produced in the decay of the lightest right-handed neutrino[10]:

$$
\left|\varepsilon_{N_{1}}\right| \leq \frac{3}{16 \pi} \frac{m_{N_{1}}}{v^{2}} m_{v_{3}} .
$$

and the final lepton asymmetry is given by

$$
Y_{\ell}=\eta \varepsilon_{N_{1}}
$$


where $\eta$ is the efficiency parameter which is controlled by the washout regime. These are the two main parameters that determine the final value of the lepton asymmetry.

Spectator Effects We consider processes in the thermal bath of the early Universe that conserve $B-L$. For a given temperature $T$, from chemical equilibrium considerations we require: Total isospin, hypercharge and color must be zero, flavor changing interactions involving the quarks are in equilibrium, Yukawa interactions are equilibrium, electroweak and QCD sphalerons are in equilibrium, and in a supersymmetric model additionally particles in same multiplet have $\tilde{\phi}=\phi$. Thus, we can then solve for $B, L, B-L$ in terms of a chemical potential, say $\ell$.

\section{Lepton Flavour Issues}

Given that $h_{e}, h_{\mu}, h_{\tau}$ are small and the lepton asymmetry is obtained from a trace in flavour space how can flavour matter? However, as flavour distinguishes mass eigenstates, this has some unforeseen consequences [7]. For temperatures where $\Gamma_{\tau}, \Gamma_{\mu} \gg H$ then it is cleat that the charged lepton Yukawas have no effect of the CP asymmetry but the dynamical process of right-handed neutrinos decay and production and the corresponding lepton asymmetry is distributed among distinguishable flavours. In this way, we see that charged lepton Yukawa interactions have the effect of projectors, asymmetries in each flavour are washed out differently, contributing with different weights in the expression for the total asymmetry.

\subsection{Purely Flavour Leptogenesis}

In this type of models it is possible to generate the baryon asymmetry even in the case in which $\sum_{\alpha} \varepsilon_{\alpha}=0$ without lepton number violation in decay [12]. In this case the Boltzmann equations for lepton flavour asymmetries in general present different washouts in the different flavours so..... $Y_{B-L} \propto \sum \eta_{\alpha} \varepsilon_{\alpha} \neq 0$. We rely on the condition that the dynamics of the different lepton flavors are decoupled at the leptogenesis temperature. Most importantly, $\mathrm{CP}$ violation can originate from the non leptonic sector/lepton number conserving loop. A specific case is a model with lepton flavour violation $(L F V)$. There is no need to have lepton number violation in the loop, lepton number violation only via the washout processes.

\subsection{Lepton Flavour Equilibration}

Most extensions of the SM, and most noticeably among these

the Supersymmetric Standard Model (SSM), include new sources of Lepton Flavor Violation (LFV). If leptogenesis occurs at temperatures when these new sources mediate reactions that are in chemical equilibrium, then there are no flavor effects in leptogenesis. With Lepton Flavor Equilibration (LFE) [18], we refer to the effect of reactions that would bring the different lepton doublets $\ell_{\alpha}(\alpha=e, \mu, \tau)$ into chemical equilibrium. The main points to keep in mind are:

- In the presence of LFV, fast $\ell_{\alpha} \rightarrow \ell_{\beta}$ transitions effectively eliminate all dynamical flavor effects.

- If at $T_{L G}$ the LFV processes are in chemical equilibrium then there are no flavour effects and the one-flavour approximation correctly describes the production of the lepton asymmetry. 
- Must be included in the chemical equilibration/BEs and Spectator processes become more relevant.

- In general, models of new physics have new sources of LFV, so it is not immediate that flavour effects will survive.

We will use as a general and most interesting example the SSM where, in the basis in which the charged lepton Yukawa couplings are diagonal, a source of LFV from soft supersymmetry breaking masses is generally present:

$$
\mathscr{L}_{\text {soft }} \supset \tilde{m}_{\alpha \beta}^{2} \tilde{\ell}_{\alpha}^{\dagger} \tilde{\ell}_{\beta}
$$

Here $\tilde{\ell}_{\alpha}$ are the superpartners of the $S U(2)$ lepton doublets. The terms in 2.1 affect the flavor composition of the mass eigenstates, and as a result the $\ell_{\alpha} \tilde{\ell}_{\alpha}^{(\text {int })} \tilde{G}$ vertex for the sleptons gauge eigenstates (where $\tilde{G}=\tilde{W}_{a}, \tilde{B}$ represent a $S U(2)$ or $U(1)$ gaugino) involve a unitary rotation to the slepton mass eigenstates:

$$
\tilde{\ell}_{\alpha}^{(\text {int })}=R_{\alpha \beta} \tilde{\ell}_{\beta}, \quad R_{\alpha \beta} \sim \delta_{\alpha \beta}+\mathscr{O}\left(\frac{\tilde{m}_{\alpha \beta}^{2}}{h_{\alpha}^{2} T^{2}}\right),
$$

where $h_{\alpha}>h_{\beta}$ is the relevant charged lepton Yukawa coupling that determines at leading order the (thermal) mass splittings of the sleptons.

A supersymmetric model with soft supersymmetry-breaking mass terms $m_{\alpha \beta}^{2}$ gives rise to new diagrams that could contribute to a lepton asymmetry via PFL à la soft leptogenesis.

- Flavour $\mathrm{CP}$ asymmetries which are enhanced as $\propto g^{2} \lambda$, but $\varepsilon=\sum_{\alpha} \varepsilon_{\alpha}=0$.

- However, these are ineffective because lepton flavour densities equilibrate very fast, so ...LG described by single flavour approximation, which cannot be sourced by the type of diagram above.

\section{Conclusions}

Extensions of the Standard Model can provide the necessary ingredients for successful implementation of the leptogenesis mechanism. A careful treatment of the lepton flavour basis in relevant for the final quantitative results. Although detailed quantitative results can be obtained the more difficult aspect of the leptogenesis mechanism is currently its testability.

\section{References}

[1] M. Fukugita and T. Yanagida, Phys. Lett. B 174, 45 (1986). M. A. Luty, Phys. Rev. D 45, 455 (1992).

[2] For recent reviews see: W. Buchmuller, R. D. Peccei and T. Yanagida, Ann. Rev. Nucl. Part. Sci. 55, 311 (2005) [arXiv:hep-ph/0502169].

S. Davidson, E. Nardi and Y. Nir, Phys. Rept. 466, 105 (2008); arXiv:0802.2962 [hep-ph]. A. Strumia, arXiv:hep-ph/0608347; E. Nardi, arXiv:hep-ph/0702033; Y. Nir, arXiv:hep-ph/0702199; M. C. Chen, arXiv:hep-ph/0703087; E. Nardi, arXiv:0706.0487 [hep-ph]; A. Pilaftsis, arXiv:0904.1182 [hep-ph].

[3] A. D. Sakharov, Pisma Zh. Eksp. Teor. Fiz. 5, 32 (1967) [JETP Lett. 5, 24 (1967)]. 
[4] P. Minkowski, Phys. Lett. B 67421 (1977); T. Yanagida, in Proc. of Workshop on Unified Theory and Baryon number in the Universe, eds. O. Sawada and A. Sugamoto, KEK, Tsukuba, (1979) p.95; M. Gell-Mann, P. Ramond and R. Slansky, in Supergravity, eds P. van Niewenhuizen and D. Z. Freedman (North Holland, Amsterdam 1980) p.315; P. Ramond, Sanibel talk, retroprinted as hep-ph/9809459; S. L. Glashow, in Quarks and Leptons, Cargèse lectures, eds M. Lévy, (Plenum, 1980, New York) p. 707; R. N. Mohapatra and G. Senjanović, Phys. Rev. Lett. 44, 912 (1980).

[5] V. A. Kuzmin, V. A. Rubakov and M. E. Shaposhnikov, Phys. Lett. B 155, 36 (1985).

[6] A. Pilaftsis, Phys. Rev. D 56, 5431 (1997) [arXiv:hep-ph/9707235].

[7] A. Abada, S. Davidson, F. X. Josse-Michaux, M. Losada and A. Riotto, JCAP 0604 (2006) 004 [arXiv:hep-ph/0601083]. E. Nardi, Y. Nir, E. Roulet and J. Racker, JHEP 0601 (2006) 164 [arXiv:hep-ph/0601084].

A. Abada, S. Davidson, A. Ibarra, F. X. Josse-Michaux, M. Losada and A. Riotto, JHEP 0609 (2006) 010 arXiv:hep-ph/0605281.

R. Barbieri, P. Creminelli, A. Strumia and N. Tetradis, Nucl. Phys. B 575, 61 (2000) (for the updated version of this paper see [arXiv:hep-ph/9911315]).

[8] A. Pilaftsis and T. E. J. Underwood, Phys. Rev. D 72, 113001 (2005) [arXiv:hep-ph/0506107]. T. Endoh, T. Morozumi and Z. h. Xiong, Prog. Theor. Phys. 111, 123 (2004) [arXiv:hep-ph/0308276];

[9] K. Hamaguchi, H. Murayama and T. Yanagida, Phys. Rev. D 65, 043512 (2002) [arXiv:hep-ph/0109030].

[10] S. Davidson and A. Ibarra, Phys. Lett. B 535, 25 (2002) [arXiv:hep-ph/0202239].

[11] M. Plumacher, Z. Phys. C 74, 549 (1997) [arXiv:hep-ph/9604229].

[12] D. Aristizabal Sierra, M. Losada and E. Nardi, Phys. Lett. B 659, 328 (2008) [arXiv:0705.1489 [hep-ph]].

[13] J. Racker and E. Roulet, JHEP 0903, 065 (2009) [arXiv:0812.4285 [hep-ph]].

[14] T. Hambye, Y. Lin, A. Notari, M. Papucci and A. Strumia, Nucl. Phys. B 695, 169 (2004) [arXiv:hep-ph/0312203].

[15] T. Hambye, M. Raidal and A. Strumia, Phys. Lett. B 632, 667 (2006) [arXiv:hep-ph/0510008].

[16] For review of neutrino properties, see C. Amsler et al. [Particle Data Group], Phys. Lett. B 667, 1 (2008).

[17] A. Ibarra and C. Simonetto, arXiv:0903.1776 [hep-ph].

[18] D. Aristizabal Sierra, M. Losada and E. Nardi, arXiv:0905.0662 [hep-ph].

[19] M. L. Brooks et al. [MEGA Collaboration], Phys. Rev. Lett. 83, 1521 (1999) [arXiv:hep-ex/9905013]. M. Ahmed et al. [MEGA Collaboration], Phys. Rev. D 65, 112002 (2002) [arXiv:hep-ex/0111030]. 